\title{
Dumb pumps, smart flow, and a genius
}

\author{
Ashish S. Shah, MD
}

From the Department of Cardiac Surgery, Vanderbilt University Medical Center, Nashville, Tenn. Disclosures: Author has nothing to disclose with regard to commercial support.

Received for publication Feb 15, 2017; accepted for publication Feb 15, 2017; available ahead of print June 20, 2017.

Address for reprints: Ashish S. Shah, MD, Vanderbilt Medical Center East, 1215 21st Ave South, Suite 5025, Nashville, TN 37232 (E-mail: ashish.s.shah@vanderbilt.edu).

J Thorac Cardiovasc Surg 2017;154:879

$0022-5223 / \$ 36.00$

Copyright (C) 2017 by The American Association for Thoracic Surgery

http://dx.doi.org/10.1016/j.jtcvs.2017.02.025

In 1513, Leonardo da Vinci conducted a series of experiments to understand how blood flows through the aortic root and influences the motion and function of aortic valve leaflets. ${ }^{1}$ With the use of grass seeds in glass aortic root molds, da Vinci's elegant and brilliant approach illuminated the complex physiology of the aortic root. Of note, he established that blood flow influences the conduit. The present work by Yoshida and colleagues ${ }^{2}$ recreates this experiment using echocardiography and computational flow dynamics to characterize the complex relationship among the native blood flow, mechanical blood flow, and aortic root. The study highlights the possibilities of modern imaging and computational methods to help us understand the inconvenient truth that pushing blood to help failing hearts is an imperfect solution. Multiple left ventricular assist device (LVAD) complications have emerged to prevent truly widespread adoption. In particular, vascular complications, stroke, arteriovenous malformations, bleeding, and progressive aortic insufficiency have plagued investigators and clinicians. As highlighted by the current study, one possible source may be the flow characteristics in the aortic root itself. Blood is a complex fluid, and because of its mass creates interesting flow dynamics particularly at the boundary layer, that is, the interface between the fluid and the vessel wall. The study by the Osaka group demonstrates that early after LVAD support there are unique stresses on the aorta and the aortic valve. What is unclear is how this changes over time and, importantly, whether those changes are predictive of future complications. Although the patient in this case had an ischemic cardiomyopathy, she was only 43 years old. How would flow look in a 60- or 70-year-old aorta? Does the cause of heart failure matter? Do the different device designs have similar flow characteristics and does the angle of the outflow graft play a role in aortic flow dynamics? Ambardekar and colleagues ${ }^{3}$ have reported significant changes in aortic compliance after LVAD placement. They go on to speculate that there may an association with these changes and ongoing myocardial load and

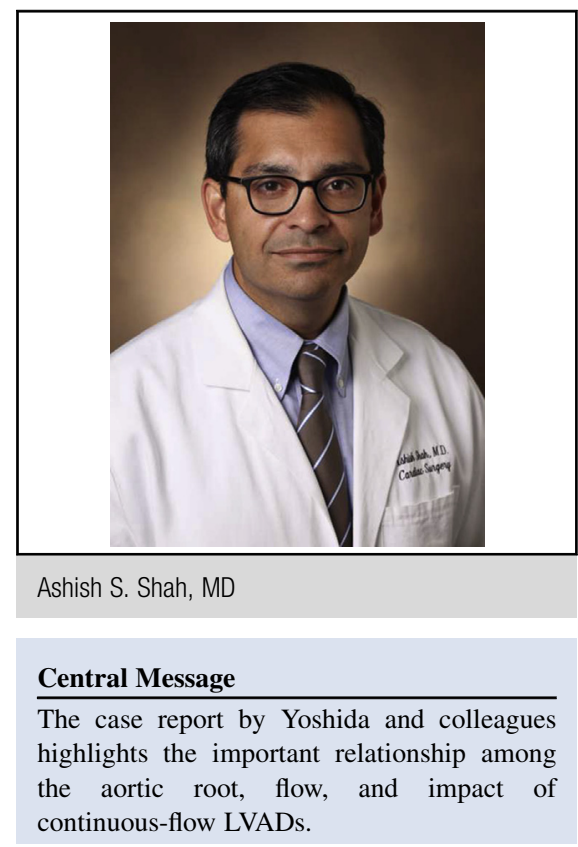

See Article page 877.

stress. $^{3}$ There is also an emerging connection between genetically triggered aortopathy and heart failure. ${ }^{4}$ Therefore, it is intriguing to think about this interaction among flow in the aortic root, peripheral vasculature, and genetic maladaptive remodeling. Moreover, it suggests that how we propel blood as a function of time during the cardiac cycle may be a critical regulator of device biocompatibility. Perhaps pulsatility has a purpose after all. Yoshida and colleagues ${ }^{2}$ have revisited Leonardo's extraordinary experiments and in turn have started an important line of inquiry that should challenge investigators and our industry partners to evolve these devices away from "dumb pumps" to respectful and physiologic ones.

\section{References}

1. Robicsek F. Leonardo da Vinci and the sinuses of Valsalva. Ann Thorac Surg. 1991:52:328-35.

2. Yoshida S, Fukushima S, Miyagawa S, Toda K, Sawa Y. Visualization of vortex flow and shear stress in the aortic root during left ventricular assist device support. J Thorac Cardiovasc Surg. 2017;154:877-8.e1.

3. Ambardekar AV, Hunter KS, Babu AN, Tuder RM, Dodson RB, Lindenfeld J. Changes in aortic wall structure, composition, and stiffness with continuousflow left ventricular assist devices: a pilot study. Circ Heart Fail. 2015;8:944-52. 4. Haldar SM, Lu Y, Jeyaraj D, Kawanami D, Cui Y, Eapen SJ, et al. Klf15 deficiency is a molecular link between heart failure and aortic aneurysm formation. Sci Transl Med. 2010;2:26ra26. 